\title{
Supporting agricultural policy - the role of scientists and analysts in managing political risk
}

\author{
K.B. Matthews ${ }^{\mathrm{a}}$, D.G. Miller ${ }^{\mathrm{a}}$ and D. Wardell-Johnson ${ }^{\mathrm{a}}$ \\ ${ }^{a}$ James Hutton Institute, Aberdeen, United Kingdom \\ Email: keith.matthews@,hutton.ac.uk
}

\begin{abstract}
This paper reflects on the role of scientists within research organisations and analysts within the Scottish Government in managing political risk for major policy changes. The particular change considered is the reform of direct payments to farmers through Pillar 1 of the Common Agricultural Policy, moving from payments based on historic entitlements to a multi-regional, area-based payment scheme. The move in Scotland to such payments is a major change in policy and one likely to result in significant redistribution in direct payments that underpin the financial viability of many businesses. The policy change thus involves considerable risk for stakeholders within the agri-food system but also politically for the Scottish Government for whom agriculture is an area of devolved responsibility. Considerable uncertainty surrounds the decision making process, partly as a result of EU processes requiring both EU Parliamentary and Member State agreement. Furthermore, the Scottish Government recognises and wishes to strike a balance between food production and associated economic activities conducted mainly in lowland areas and the ecosystem services delivered in the main by upland areas. Limited timescales for implementing the new policies also mean that research often needs to be undertaken before final decisions are made at EU and UK level.
\end{abstract}

The uncertainty in policy objectives and the spatial heterogeneity of Scotland's agricultural systems also mean that the policy options cannot easily be subjected to simple, single objective cost-benefit analysis. This policy uncertainty combined with a desire for the process of analysis to be transparent and inclusive meant that multiple scenarios, performance metrics and summaries were required by Scottish Government. The paper presents examples of the most important outputs for the spatial analysis framework and how these were used. Over the course of the research, it has become increasingly clear that political risk management in a complex and uncertain environment strongly shapes both the timing and use of research-based analysis. The paper shows how the research contributed to this risk management strategy by quantifying uncertainties, testing scenarios and communicating with stakeholders both formally and informally.

The paper concludes that the use of a spatial analysis framework was effective in highlighting the most significant redistribution effects - particularly those that occur within sectors or regions. These were useful to stakeholders in helping them articulate to government the likely adaptive responses from farming systems and to give an impression of the wider consequences for rural communities and the natural environment. From the experiences of working across the science-policy interface the authors conclude that simplistic structural models of science-policy interactions fail to provide diagnostic information needed to improve outcomes since they fail to represent the messy process of science-policy interaction. This process is dependent on the ability of individuals from either side of the science-policy interface to form mutually beneficial partnerships without compromising their independence. This network building is facilitated by the aim of the authors' research organisation to function as a boundary organisation, facilitating exchanges between science and policy and by the openness to close cooperation of individual analysts within Scottish Government analysis teams. There are significant differences in research cultures but sufficient common ground in terms of disciplinary skills, ontologies and epistemologies and common cause in tackling challenging policy risks. This capacity for cooperation is activated by Scottish Government funding models that support the long term development of strategically relevant research capacity, the building of networks and social capital bonds between analysts and scientists and additional flexible funding that can be deployed quickly by policy teams to address immediate questions. Within this environment, scientists can have a key role in bringing innovations into the analysis conducted in support of risk management, since their independence means they have greater freedom to work across Scottish Government departmental boundaries and within Scottish Government hierarchies. Success in this role, however, depends on institutional support for process wherein scientific credibility is translated into policy credibility incrementally through demonstration of salience, timeliness and adaptability.

Keywords: science-policy, risk management, spatial analysis, research culture, common agricultural policy 


\section{INTRODUCTION}

\subsection{Policy Background}

The Common Agricultural Policy (CAP) is the oldest common policy of the European Union dating conceptually from the treaty of Rome in 1957, with first implementation in 1962. Its stated objectives, their balance and mechanisms have continually evolved but continue to encompass income support for farmers (70\% of budget), rural development $(20 \%)$ and market support $(10 \%)$. Since 2000 , there has been increasing pressure to reform the CAP both from the World Trade Organisation and some Member States (MS). Newer MSs prefer to see support directed through structural funds to other sectors of their economies and there is also a desire by some MSs to emphasise provision of non-market or ecosystem goods and services. In 2000 the CAP was divided into two Pillars - the first for direct support and the second for rural development. In 2003, payments to farmers were "decoupled" from the specifics of production with the intention that subsidy would no longer distort management decisions and that EU farming would become more market oriented and thereby WTO compliant. Associated with this change was the stated intention to simplify and regularize payments by paying a standard "flat" rate for all eligible land within a region and that over time this rate would converge between MSs. MSs, however, retained the option of continuing to make payments based on entitlements between 2000 and 2002 (the historic model); moving to area-based payments or having a hybrid of partly historic and partly area-based. UK-Scotland adopted an historic model with decoupling. The European Commission position was, however, that the 2003 arrangements were for a transition period and that post 2013 area based payments would be introduced without exception. This provided an exceptionally long window within which to prepare for an area-based system (yet with remaining uncertainty of its implementation). The Scottish Government commissioned an independent inquiry in 2010 into future support for agriculture (the Pack Inquiry) to inform decision makers of the issues and the range of options available. The Pack Inquiry was supported both by the authors' research team and by analysts within the civil service in Scotland. Consultation by the EU on the post-2013 CAP began with draft proposals from the EC in 2011 followed by two years of negotiations between EC, MS representatives through the Committee on Agriculture or Heads of Government and for the first time the European Parliament. This complexity of negotiation is added to by the autonomy given to regions within MS on how the regulations are implemented.

\subsection{Research Background}

Research in support of Scottish Government policy is provided from within government by the Rural and Environmental Science and Analytical Services Division (RESAS) (the analysts) and from the Main Research Providers (the scientists) who undertake research programmes managed by RESAS. These programmes undertake both strategic and applied research and scientists within MRPs are increasingly involved in policy-led research where questions are defined by policy needs rather than curiosity-led. The research team supporting policy development in this area has a long history of policy-oriented work. The policy topics have included climate change impacts, previous CAP reforms, the justification of payment rates for agri-environment payments and greenhouse gas emissions from land use change. The approach to such topics has, however, evolved from case-study based analysis using decision support systems (Matthews et al., 2007), into the design and implementation of spatial analysis frameworks with national coverage but field scale granularity. This granularity of analysis and implementation is necessary to cope with the heterogeneity of Scotland's land use systems. These spatial analysis frameworks integrate data from administrative sources (e.g. annual land ownership, user-ship, use and cover) and from research (e.g. land capability for agriculture, soils and climate). There has been a focus on the integrated use of these datasets to generate mapped secondary variables such as stocking rates. During the course of this research, the team have also developed skills in the organisation and execution of deliberative inclusive processes wherein the outputs of research are subject to scrutiny by stakeholders and policy makers and knowledge elicited from such events is incorporated into the analysis or its presentation. The research presented in this paper encompasses three phases - 1) strategic capacity building and prototyping (2008-9), applied in support of the Pack Inquiry (2010) and policy-led (2011-12) directly in support of the Scottish Government policy teams.

\subsection{Managing Political Risk}

The uncertainties engendered by multi-level governance within EU processes, the desire by the Scottish Government to strike a balance between production and ecosystem service provision and the time constraints between agreement of regulations and their implementation create political risks both in terms of the eventual outcomes (policy or policy delivery failure) and the legitimacy of how the process of policy development is carried out. Legitimacy hinges on the degree to which stakeholders feel that their voices have been heard and 
on the fairness and impartiality of the evidence used as a basis for decision making. In this regard science and scientists need to guard against being used as a means of supressing alternative discourses rather than helping to appropriately structure and support policy formulation (Wesselink et al., 2013). The political risks of implementing CAP reforms are substantial despite the small size of the sector in terms of gross value added or employment. While the bulk of CAP spending of 642M€ is conventional income support there are many other agri-environment, community and rural development programmes that depend on the associated funding. Further, agricultural, sporting and forestry management still accounts for the vast majority of land use in Scotland (>90\%). The safety, security, quality and affordability of food are argued, in stakeholder discourse, as being tied to agriculture policy, as is character of rural landscapes within which a substantial proportion of the population live or use for recreation. Stakeholders in the area are also well organised, vociferous and well-connected politically.

The primary role in managing these political risks falls to the policy teams within the Scottish Government drawing on work by in-house analysts, but also increasingly with an expectation of inputs from scientists funded within the Scottish Government strategic research programmes. In this context, interaction between research scientists and policy teams is mediated by analysts within RESAS who act as gatekeepers. These individuals conduct research in their own right and share ontologies, epistemologies, disciplinary and technical skills as scientists. Yet analysts operate in a completely different research culture. This is most easily seen in the timescales over which research is conducted, but perhaps more significant are the greater requirements to operate within formal and hierarchical structures of decision making. There are thus two communities of practice separated by research cultures.

Recognising this difference in cultures, the paper therefore addresses the question, what are the necessary procedural and institutional conditions needed to ensure that the outputs from scientific research play a positive role in the political risk management for major policy changes? The paper argues that the interface between science and policy need not be an impenetrable barrier or dead-zone gap, yet cannot be assumed to be an imperceptible seamless transition. The paper argues that there are significant differences and that these require individual scientists and analysts to devote time and resources to overcoming them. For this to happen there needs to be an institutional framework in place that supports and rewards science-policy interactions (for both sides), a permissive attitude to informal contact and long-term funding of strategic research that later underpins the capacity to respond to immediate policy-led needs. The outcome of more frequent policy-oriented interactions is a better understanding by scientists of the constraints within which analysts and policy makers operate. Better understanding of the policy and analysis institutional and governance environment enhances the ability of scientists to anticipate policy needs, design analyses, tailor and communicate outputs in a way that they are immediately salient for political risk management. Finally, a longitudinal commitment to such activities by scientists is essential to support the incremental and iterative process of translating scientific credibility into political risk management credibility.

\section{MATERIALS AND METHODS - SCIENCE CONTENT}

\subsection{Regions and Budgets}

The research supports policy decisions on the implementation of the post-2013 CAP in Scotland by reviewing alternative options for Pillar 1 CAP payments in Scotland and assessing their distributional consequences. All the uncertainties highlighted above make satisfying all stakeholders challenging, with political risks even in the choices of parameters or weightings. Two key decisions are how to divide Scotland into different payment-regions and what share of the budget to allocate to each region. Payment rates are set by dividing budgets by area of payment regions. Two regionalisation options with ten variants were tested within the Pack Inquiry analysis. The payment-regions were defined by Land Capability for Agriculture (LCA) classes $^{1}$ or Less Favoured Area (LFA) an administrative classification used to define areas handicapped by their poor quality natural resources and/or remoteness. In follow up work, both these options were continued with two, three and four payment-region LCA based options considered, a new three payment-region LFA and a three payment-region classification based on land use (arable including temporary grasslands, permanent grasslands and rough grazing). In all these cases, each farm business is made up of a mix of the payment-regions defined by overlaying maps of the field boundaries of lands used with the payment-region maps. The number of regionalisation options was further increased by consideration of classifying agricultural parishes rather than farms using variants of the farm level regions (plus a classification based on the average rates of receipts under the existing scheme). All told 10 regionalisation options were considered.

\footnotetext{
${ }^{1}$ A 13 zone classification where each class is made up of multiple non-contiguous parcels of land.
} 
In the 2010 Pack Inquiry, modelled payment rates were set through minimising the amount of redistribution between recipients within the overall budget constraint. For the policy-led analysis in 2011-12, the setting of budgets had to be objective and non-discriminatory with defined mechanisms for allocating funds. Three options were tested: historic, economic and land area weighted. Historic budgeting used current entitlements per region (as defined in 2003, but in some cases traded between businesses). Since it is not straightforward to determine which land within farms generated previous entitlements, it was necessary to compromise and average payments across all land within a business was used, inflating rates on the poorer quality land and depressing them on higher quality land. For the economic budgeting, the financial contribution of the enterprise mix on each business in 2011 was assessed (using the value of outputs at the farm gate). Again, within businesses it was not possible in all cases to definitively link these values to land within the holding, with aver ageing increasing the rates of payment set for lower quality land. For land-area weighted rates the areas of each payment-region were weighted so as to achieve the desired relativities of spend and hence payment rates. Since weightings are used, the policy decision is no longer only the basis of budgeting (e.g. historic or economic) but the choice of land-based weights to be used. Two variants were considered. One used the weights to ensure that the highest rates of payment occurred on mid-grade land, with best land receiving a $20 \%$ lower rate than the mid-grade and the poorest land a fixed rate of $27 €$ per ha. The intent was to support farming systems important to the agri-food supply chain and vulnerable to reduced support. The second increased payment to the poorest land by 10\% compared with historic rates and constrained the best rates to be no more than twice that on the mid-grade land. This weighted payment towards land more strongly associated with the provision of ecosystem goods and services. Not all regions options were combined with all budgets as this would have generated 40 scenarios - the production and environmental weightings were applied to a subset of regions (30 scenarios).

Note that for policy development all other factors were as far as possible held constant so that the effects of the decisions on regions and budgets could be understood in isolation. These included eligible area (defined by land use, intensity of use and previous entitlements ${ }^{2}$ ), the rate and timing of conversion to area payments (100\% area-based was modelled as the end point), overall budget (no new/small farmers schemes, or production coupled support - likely to be $7-12 \%$ of budget to allow full like for like comparisons).

\subsection{Metrics and Evaluations}

The outputs from the spatial analysis framework are the change in payments for each business $(18,790$ in 2011). The sum of the absolute value of these increases and reductions is a key metric since it defines the redistribution between existing recipients (the welfare effect). The lowest value for redistribution for the scenarios considered is $267 \mathrm{M} €$ or $42 \%$ of the budget ${ }^{3}$. The magnitude of this redistribution reflects the considerable differences between businesses in historic subsidy rates that were linked to production intensity. These differences are "flattened" out by the use of area-based payments where rates of payment depend on the regionalisation and budget decisions, not intensity of use. In addition to the magnitude of the redistributive effects, the number of businesses experiencing increases or decreases was seen to be a crucial metric in assessing the likely acceptability of any proposal. Policy makers are somewhat sanguine about the need for redistribution, especially since in some cases there are concerns about high value entitlements being activated with minimal activity on poor quality land. Some sectors were also perceived by the Pack Inquiry as having been over-supported in previous CAP regimes. Set against this is the need to ensure that the changes did not result in significant reductions in production that could undermine key sectors or regions. This was seen as particularly important for livestock raising businesses that provide inputs to the finishing and processing industries and the wider agri food chain. Set against this is the need to ensure that businesses that, while marginal economically, are delivering significant ecosystem goods (habitats etc.) are more adequately rewarded by the new CAP than the previous regime oriented to production or maintaining rural populations. The headline levels of redistribution were sufficiently large that further analyses were required to assess which sectors and regions were being most affected.

Each business was assigned to a sector based on the majority ( $66 \%$ by value) of its outputs (this gave 23 sectors). Businesses were also assigned a regional identifier based on where the largest share of its land occurs. With these classifications it was possible to generate regional and sectoral summaries both in terms of the net effects per region/sector but also the magnitude of increase and reductions. A further summary of the outcomes using six size classes (between $<50$ ha and $250+$ ha) was intended to assess the distribution of increases and reductions and the relative burden sharing.

\footnotetext{
${ }^{2}$ Each of these has the potential to increase or decrease the overall area on which payment is made.

${ }^{3}$ Note that if new land was included post 2013 this would an at best fixed budget over a larger area so that losses would be larger and gains less to fund those businesses without previous entitlements.
} 


\section{RESULTS - COMMUNICATING COMPLEXITY}

\subsection{Examples of Using the Modelling Outputs}

A challenge for the research was to present the outputs from the modelling in ways that were rigorous but at the same time comprehensible to policy makers and key stakeholders. High level summaries comparing the scenarios (combination of region and budget) used metrics such as overall levels of redistribution, counts of businesses receiving increased or reduced payments, budgets and payment rate per region. These summaries were supported by characterising how the distribution of expenditure changes ${ }^{4}$. Figure 1 , shows two example outputs used with stakeholders and policy teams. The left part shows the increases and reductions by sector both as Euro totals and as counts of businesses. The elements are ordered using net redistribution from most negative to most positive. The sector that is highlighted is Specialist Beef - which experiences a neutral net redistribution but significant within sector redistribution from more intensive businesses to less intensive.

Communicating the pattern of increases and reductions was prioritised as these are what are experienced by individual businesses. The balance between increases and reductions is only implied by the differences between the bars and the ordering of elements (higher net reductions to neutral to higher net increases). With individual scenarios this is highly effective in communicating where the greater net effects occur, yet with hindsight a fixed ordering could have been preferable to make comparison between scenarios simpler.

Characterising within sector redistribution rather than net effects is probably the most important outcome of the research in terms of influencing the scenario evaluation methodology within government. It demonstrates conclusively that while net effect can be very small, this can disguise very substantial redistribution within sectors or regions and that distribution effects cannot be safely ignored. The information also shaped the stakeholder debate in that discussion included effects within particular sectors rather than the balance of resources between sectors. The justification for production-coupled support in sectors judged to have excessive within sector redistribution could also be made with indicative magnitudes assessed.

Both regions and sectors are complex figures but each (and a similar size based comparison) uses the same visual grammar in the same way to enhance comprehension. Experience with previous projects meant that complex illustrations were not rejected per se but needed careful initial explanation of the visual grammar so that its content could then be readily understood. The assumption here was that in the main the key use of these materials would be in interactive fora where the research team could assist in interpretation.

\subsection{Process - actors and networks}

Given the magnitude and complexity of change combined with the uncertainty in the framing of the new regulations, there was considerable opportunity for suggestion of options to explore both within government and between government and stakeholders. The process of assessing and prioritising options to be assessed is mediated by the policy leads within government in consultation with, and ultimately by ministers. There are a plurality of forums and communications channels some direct and others mediated and others highly informal yet strongly influential. This decision making environment is at odds with a simple model of science-policy interactions where transactions are conducted across a well-defined boundary with a purchaser-provider relationship between policy-makers and researchers. Key relationships for the research team were with the government analysts since they share similar technical expertise and appreciation of the difficulty and resource needs of analysis. The analysts were initially the gate keepers and facilitators for the research team, but over time the research team built their own direct linkages with policy delivery teams who could provide data and assess implementation feasibility. Yet with the policy teams the analysts have also continued to act as intermediaries or partners being present in nearly all interactions. These interactions are organised within a series of fora with a hierarchy, many of which the research team were either formally or informally part of. Interactions with stakeholders have occurred through formal government-organised fora - the Future CAP Stakeholders Group and conferences but also informally through invitations to present or discuss results at meetings of the National Farmers Union (Scotland) board. In these fora it is possible for the research team to gain insights into stakeholder concerns and to undertake supplementary analyses to investigate and articulate particular issues or suggest their inclusion to government. In this regard a strategic research programme gives flexibility for the research team to undertake analyses beyond the scope of the policy-led work and on occasion these have subsequently been incorporated into the formal Scottish Government analysis process.

\footnotetext{
4 All scenarios are set out in http://www.scotland.gov.uk/Topics/farmingrural/Agriculture/CAP/regulations/Meetings-2013/Future-CAPScenarios-Analysis-Summaries
} 

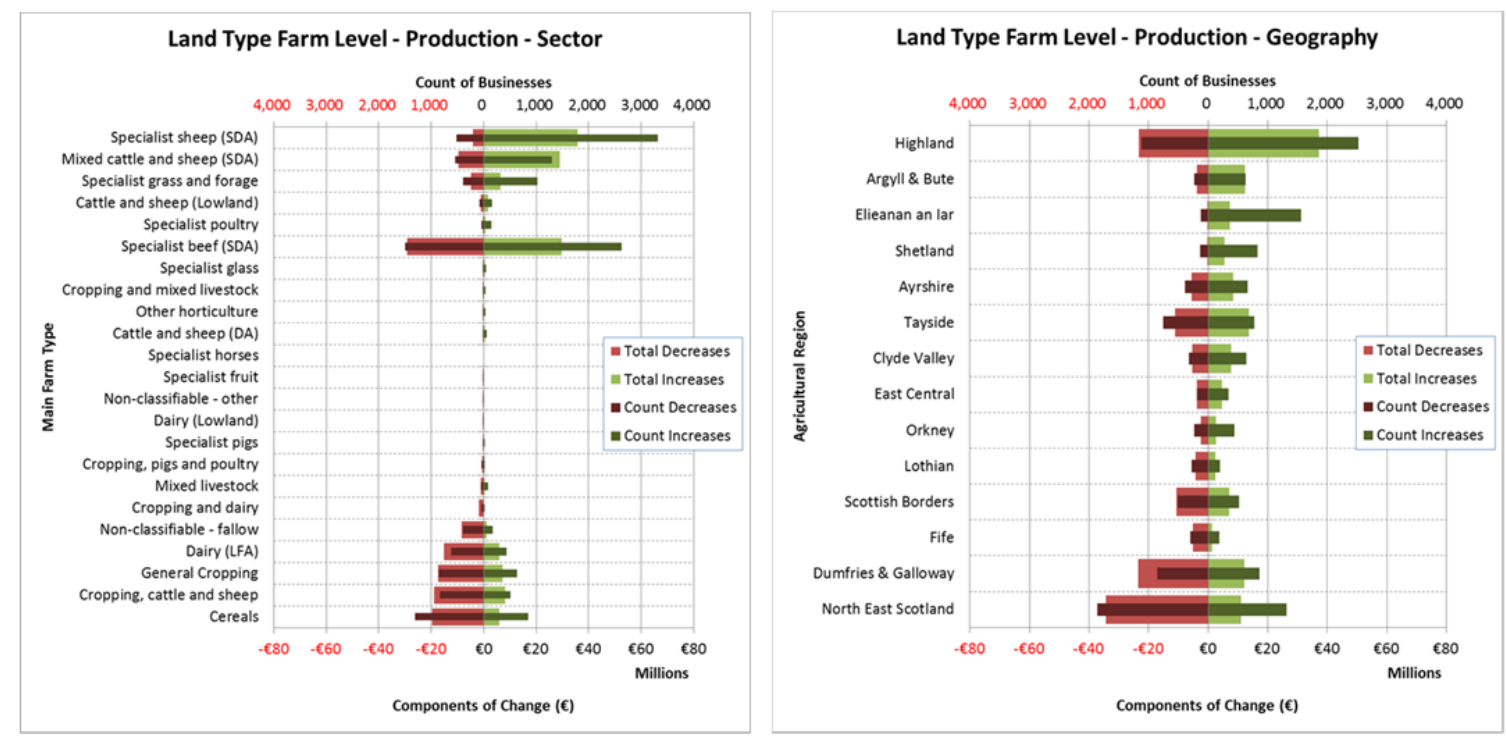

Figure 1, Example outputs from the region and budget scenario analysis

\section{DISCUSSION AND CONCLUSIONS}

Political risk management. The changes in the CAP from 2014 are a source of political risk for the Scottish Government. Managing those risks means they need to be proactive in undertaking timely analysis to underpin their negotiating positions within the EU and UK dialogues. Additionally, they need to be concerned with the cost and ease of implementation of any new CAP system. The development of policy positions requires making multi-objective trade-offs in a highly complex and uncertain decision space. Uncertainty stems from both the lack of specificity on the eventual shape of the agreement and from the adaptive response from land managers that will shape the wider socio-economic and environmental impacts of the change. Together these severely limit the scope for a conventional options appraisal. The research team could, however, provide a spatial analysis framework within which important components of the future CAP system could be tested in terms of their effects on the distribution of support across sectors, regions and business sizes. Distributional effects enable stakeholders to better articulate the likely adaptive responses compared with net outcomes or averaged changes that subsume gains and losses. They also contribute to the degree of transparency in policy development and to some degree enhance the legitimacy of the process.

Models of the Science-Policy Interface. Simplistic models of science-policy interactions that focus on idealised flows of information (as a commodity) through formalised channels are not effective in ensuring that relevant research outputs are generated and used decisively in political risk management. While such models may identify what is desired, they fail to address mechanisms, transaction costs and institutional or cultural barriers (Matthews et al., 2011). They thus fail to provide diagnostic evidence for iterative improvement. Their failure can in part be explained by their advocates' inability to recognise or cope with the messy, pluralist, deliberative reality of policy making (Verweij et al., 2006). Indeed their author's aspiration to elegance, optimality and efficiency and emphasis on structures means that they are inflexible and thus rarely relevant to the uncertain, changing and ad hoc nature of political risk management (Wesselink et al., 2013). Better models of such systems recognise a plurality of structures and positioning options for scientists within them (Sterk et al., 2009). They also emphasise process, empowering individuals and the importance of social capital in building and maintaining resilient networks. Simplistic models also fail to emphasise the need for adaptive management and social learning which are increasingly recognised as necessary in the management of complex coupled social systems (Nelson et al., 2008).

Boundary Organisations and Research Culture. Despite these recognised challenges, operating as a boundary organisation across the science-policy interface in support of political risk management on an ongoing basis remains an aim of the authors' research organisation. This is operationalized by networks of relationship built between individual civil servants within the Scottish Government analysis division and research scientists. These relationships are built on shared identity as researchers, common skillsets and mutual appreciation of the scope of work required. Yet each live in greatly differing organisational environments with markedly different research cultures and it is this difference in cultures that constitutes a real gap between science and policy. In bridging the gap analysts bring knowledge of the formal structures 
and personalities which will influence how research outputs will be used. Scientists bring additional capabilities and capacity and greater independence of action allowing for proactive development of tools and analyses in partnership with analysts without the need for formal sign off by policy team leads. Individuals currently operating across the boundary also need to facilitate new linkages by match-making using their knowledge of research capabilities and policy needs.

Funding Institutions. The challenges of working across the science-policy interface mean that a longitudinal approach to funding is needed rather than a series of consultancies. Such a strategy is necessary to support the investment in the social capital embodied in the relationships between scientists, analysts and policy teams. It is also necessary to allow a strategic approach to research capacity development. The latter is a delicate balance between innovation led and application led work. Innovation is essential if the evidence base and approaches to policy development are to be enhanced. Yet the acid test of strategic research is that that overtime there needs to be evidence of the use of outputs or provision advice or contribution to policy debates by other means (Matthews et al., 2011). Otherwise, the research is simply curiosity-driven work more appropriately funded through other institutions with solely an innovation focus.

A key institutional innovation that has made science-policy cooperation far simpler is a form of framework agreement, where the research organisation holds part of its budget to support policy led work and this can only be released with agreement from a policy lead and sign off from the research lead that the resources are available. The cooperative agreement of these projects meant that researchers could contribute to the framing of work and the lack of formality meant that projects could practice adaptive management, refocusing resources as the research progresses or policy questions changed within the time frame of the project. While this initially provided very flexible funding for policy-led projects concerns over a cab-rank approach to use of the resources and a small overspend has led to a more formalised and cautious use of the resource.

\section{Overall Conclusions}

The research team has strong theoretical understanding and practical experience in the development of decision supporting tools and analyses and the processes of using these with stakeholders. This has translated well in terms of recognising the need to understand, and work with, existing institutions and governance arrangements (embedding) while also using the freedom of independence to suggest enhancements to analysis or linkages between policy development streams separated by inter-departmental boundaries. Salience depends on the flexibility of the tools developed (better a good answer to the right question than a perfect answer to the wrong one), expecting to have to negotiate in specification agreement, anticipating evolution of both scope and approaches needed, accepting and working within uncertainty and spending significant effort in developing effective methods of communicating complex data. The research team has, however, been fortunate to have had the opportunity to build on the credibility generated within the strategic research programme through a long period or repeated interactions with Scottish Government colleagues. This provided the opportunity to boot-strap credibility, which should also be the default for expectation for scientists working at the science-policy interface.

\section{ACKNOWLEDGEMENTS}

The research is funded by the Scottish Government through their Environmental Change Programme, Provision of Analytical Work in Support of Advice and Contract Research Fund.

\section{REFERENCES}

Matthews, K. B., Rivington, M., Blackstock, K. L., McCrum, G., Buchan, K., and Miller, D. G., Raising the bar? - The challenges of evaluating the outcomes of environmental modelling and software, Environmental Modelling and Software 26(3), 247-257, 2011.

Matthews, K. B., Schwarz, G., Buchan, K., Rivington, M., and Miller, D. G., Wither agricultural DSS?, Computers and Electronics in Agriculture 61, 149-159, 2007.

Nelson, R., Howden, M., and Smith, M. S., Using adaptive governance to rethink the way science supports Australian drought policy, Environmental Science \& Policy 11(7), 588-601, 2008.

Sterk, B., Carberry, P., Leeuwis, C., Van Ittersum, M. K., Howden, M., Meinke, H., Van Keulen, H., and Rossing, W. A. H., The interface between land use systems research and policy: Multiple arrangements and leverages, Land Use Policy 26(2), 434-442, 2009.

Verweij, M., Thompson, M., and Engel, C., Clumsy Conclusions: How to Do Policy and Research in a Complex World, In: Verweij, M. and Thompson, M. (eds.), Clumsy solutions for a complex world. Governance, Politics and Plural Perceptions, Chapter 11, 241-249, 2006.

Wesselink, A., Buchanan, K. S., Georgiadou, Y., and Turnhout, E., Technical knowledge, discursive spaces and politics at the science-policy interface, Environmental Science \& Policy 30(0), 1-9, 2013. 